psychosomatic sickness that hampers an individual's personal and professional competence, along with negatively affecting emotions, family, and social relationships. Technostress is a detrimental psychosomatic association between human beings and latest ICT; effects of which can undermine safety and wellbeing in home settings and workplace situations. The theoretical interpretation of ICT-induced stress or technostress is often poorly understood across academic, public, and policy domains. This is mainly due to the fact that technostress is a complex phenomenon involving a range of variables that vary from culture to culture including organisation's own working culture. This paper addresses this literature gap by critically examining the variables responsible for technostress in two distinct world regions: Nordic region and Indian subcontinent. In later sections, the paper discusses the implication of the findings and offers various empowering suggestions to all the players involved in technostress.

Methods Qualitative modes of inquiry including case studies are utilised in this examination to arrive at theoretical constructs, thereby drawing implications for the organisations' board and policy makers alike. The underlying premises hypothesise that the technostress severely affects individuals' wellbeing, social relationships, and offsets their productive competency, particularly at workplace.

Results Evidence indicates growing technostress in Indian subcontinent and Nordic region with increased anxiety, insomnia, psychical and mental strain, depression, and feelings of helplessness. Citizens in Nordic countries are more prone to technostress than their counterparts in Indian subcontinent because the latter suffers from the digital divide and ICT is yet not an integral component of homes and offices. Employees in IT industry often feel compulsive about delivering results in real-time without having enough time to engage in creative and sustained analysis. There are remarkable cultural differences between the two regions that resonate on personal and professional lives. Whereas electronic mails is a preferred medium of communication in Nordic region, traditional paper mail and hand written letters are still widely used throughout the Indian subcontinent with the exception of multinational companies in big cities.

Conclusions The technostress is a synthesis of complex variables working intricately together, and these factors vary in their complexity in two different cultural settings. Technostress is a growing problem which has affected Nordic region tremendously and has started to penetrate Indian subcontinent in big cities. At work places, Information Fatigue Syndrome caused by technostress is hampering concentration and professional competence of employees at a considerable rate. Technostress can be effectively dealt with creating awareness, training on ICT usage in organisations, and usage of ergonomic ICT hardware.

\section{EFFECTIVE USE OF SOCIAL MEDIA IN INJURY PREVENTION}

Joanne Banfield, Brandy Tanenbaum. Sunnybrook Health Sciences Centre, Canada

\subsection{6/injuryprev-2016-042156.1024}

Background With enough Facebook users to form the third largest country on the planet the question is not should we be using social media but rather how can we best use it. Social media is not a universal remedy for preventing injury, but it provides the opportunity for very real and untapped benefits to education, communication, and collaboration on a large-scale basis. The world of social connectedness allows for injury prevention organisations to share information, engage, ask questions, post links, videos, and much more.

Methods Explore the points where injury prevention and social media intersect. Sometimes the point is with the individual and sometimes it's with the organisations. Platforms like Facebook, Twitter, You Tube, provide the mechanism for organisations to share messages and engage their communities, but it is in knowing how to use these tools effectively and efficiently that make the difference between success and failure.

Results Participants will learn the three golden rules to social media: (1) how to listen; (2) how to engage; and (3) how to measure. In addition learning about: defining your social media strategy; thinking about conversations instead of campaigns; crafting messages that resonate; overcoming organisational challenges; and when and who to ask for help.

Conclusions Social media is today's most transparent, engaging and interactive form of public relations. It combines real time content with authentic peer-to-peer communication. Social media is not about what each one of us does or says, but about what we do and say together, worldwide.

\section{TECHNOLOGIES' USE, PERCEIVED RISKS, BENEFITS AND LAWS: A MULTIDIMENSIONAL UNFOLDING TECNIQUE}

${ }^{1}$ Veronica Diaz, ${ }^{2}$ Frank Busing, ${ }^{1}$ Maria Orozco, ${ }^{1}$ Alfredo Celis, ${ }^{3}$ Ariel Miranda ${ }^{1}$ Patricia Mendoza. 'University of Guadalajara, Mexico; ' Leiden University, The Netherlands; ${ }^{3}$ Hospital "Dr. Juan I. Menchaca", Mexico

\subsection{6/injuryprev-2016-042156.1025}

Background The use of the technologies in the everyday life brings benefits and risks, being a lot of them in home. According to the World Health Organisation, 90\% of injuries to young children occur in or around home. Burns being in this place, the most frequent injury in Mexico.

Methods Multidimensional unfolding is a technique that maps ranking data into a low-multidimensional space that allows for a visual comprehension of the data. 28 parents of Guadalajara, Mexico (15 mothers, 13 fathers), were asked about frequency of use of technologies; involving cooking equipment, light, and heat sources. Then they sorted them in a series of cards, by three different criteria: perceived risk, benefits and the necessity for law implementation in their use.

Results The most used technologies were gas stove, lighter, water heater, iron, matches and microwave. The least used technologies were oil lamp, fireworks, wood stove, gas grill and electric grill. The three perceptual maps obtained have two dimensions, which show two sets of objects each: the subjects (the sample of 28 parents) as numbers, and the objects (each technology) as labels. Parents perceived: more risks from fireworks and wood stove; most benefits from gas stove, water heater, and microwave; and the need of laws for fireworks.

Conclusions Laws are one of the most efficient ways to get people to adopt safe behaviours. Although it is important to consider that, people are more willing to accept laws for those things that they do not use or perceive as beneficial. In this case, parents did not use fireworks and also perceived them as risky without benefit and with the necessity of laws. Unlike the gas stove, being the most used, perceived with more benefits; without risks, and no need for laws. This is the first exploration in Mexico about the theme. An overwhelming majority of burns occur in house and kitchen; it requires further research about overconfidence and optimism bias around technologies. 